\title{
Cognitive control among immersed bilinguals: Considering differences in linguistic and non-linguistic processing*
}

\author{
LAURA SABOURIN \\ University of Ottawa, Department of Linguistics \\ SANTA VİNERTE \\ University of Ottawa, Department of Linguistics
}

(Received: November 01, 2017; final revision received: March 09, 2018; accepted: March 27, 2018; first published online 4 June 2018)

While several studies have shown a bilingual advantage in cognitive control, others have refuted such findings, leading to debates regarding the existence of bilingual benefits. The current study conducts two experiments to investigate this issue, focusing on the effect of the age of second language immersion in young adult non-immigrant bilinguals. We use a colour-word Stroop task to assess linguistic cognitive control, and an Attention Network Test to examine non-linguistic cognitive control. Results show significant differences between Simultaneous and Early Sequential bilinguals (typically grouped together as 'early') in the Stroop task, but these only become apparent when both languages are mixed. Simultaneous bilinguals also show improved Executive Control efficiency, particularly in the presence of alerting and orienting cues, suggesting enhanced attentional skills for this group. We discuss these findings with respect to participant grouping and task effects, noting the importance of the language environment.

Keywords: bilingual cognitive control, age of acquisition, Stroop, Attention Network Test, task effects

\section{Introduction}

Given the prevalence of bilingualism, researchers from many fields are becoming increasingly interested in the effects of knowing multiple languages on not only language processing, but also on general cognition. In this latter line of research, of particular interest has been the link between bilingualism and cognitive control - a set of processes that include, but are not limited to, inhibition, attention, conflict monitoring, selection, updating, and task-switching. Although speaking two languages has clear communicative, social, and professional advantages, whether bilingualism also confers cognitive control advantages is an ongoing debate (e.g., discussion in Cortex, 73, Della Sala \& Grafman, 2015).

With respect to bilingual language processing, the evidence favours lexical representations of both languages being simultaneously active (e.g., Green, 1998; Kroll,

\footnotetext{
* The authors thank Chantal Farhat, Natalie Ho, Joy Yu Ying Li, Sameer Ratti, and Cooper Rigg for their assistance in data collection. We also thank Christie Brien, Michèle Burkholder, members of the ERPLing $\mathrm{Lab}$ and three anonymous reviewers for feedback on several aspects of this project. Laura Sabourin was supported by a Social Sciences and Humanities Research Council of Canada Standard Research Grant and by a Social Sciences and Humanities Research Council of Canada Insight Grant, and Santa Vinerte was supported by a Social Sciences and Humanities Research Council of Canada Doctoral Fellowship and an Ontario Graduate Scholarship.
}

Bobb \& Hoshino, 2014) and shows that simultaneous activation occurs during both comprehension (Marian \& Spivey, 2003) and production (Kroll, Bobb \& Wodniecka, 2006). In other words, in addition to lexical items competing for selection within-language, this competition occurs between the two languages as well. To resolve the linguistic competition, bilinguals must pay attention to the languages in their environment, selecting the target language, inhibiting the non-target one, or rapidly switching between the two, and the same cognitive control processes are shown to be involved in both language as well as more domain-general information management. Earlier research demonstrated overlapping representations of a bilingual's languages, with dynamic control (see Abutalebi \& Green, 2007; 2008), and more recently, fMRI results have revealed a functional overlap between the neural areas involved in language processing and those used for general cognitive control (Coderre, Smith, van Heuven \& Horwitz, 2016). As a result of the interaction between networks involved in language and general control, the practice of managing two languages may strengthen cognitive control networks (Stocco, Yamasaki, Natalenko \& Prat, 2014). Although the interaction between language and cognition is complex and multifaceted (see Kroll \& Bialystok, 2013), constant use of cognitive processes for language control is suggested to lead to a general cognitive control advantage

Address for correspondence:

Laura Sabourin, University of Ottawa, Department of Linguistics, 70 Laurier Ave. E., Ottawa, ON, K1N 6N5 Canada

Laura.Sabourin@uOttawa.ca 
for bilinguals relative to monolinguals (e.g., Bialystok, Craik \& Luk, 2012).

However, the bilingual advantage hypothesis is not universally accepted, and experimental results have been inconsistent. While some studies show cognitive benefits of bilingualism (e.g., Bialystok, Craik, Klein \& Viswanathan, 2004; Bialystok, Craik \& Luk; 2008; Costa, Hernández \& Sebastián-Gallés, 2008; Pelham \& Abrams, 2014), others have noted that these effects are inconsistent (Costa, Hernández, Costa-Faidella \& Sebastián-Gallés, 2009; Hilchey \& Klein, 2011; Paap \& Greenberg, 2013; Hilchey, Saint-Aubin \& Klein, 2015). Furthermore, de Bruin, Treccani, and Della Sala (2015) report a publication bias in favour of positive results, and suggest that the bilingual advantage view may partially stem from a lack of published evidence to the contrary. Over the past few years, however, the publication trend has been changing to include a variety of outcomes, including studies that challenge bilingual benefits or are ambiguous regarding them (Sanchez-Azanza, Lópes-Penadés, BuilLegaz, Aguilar-Mediavilla \& Adrover-Roig, 2017).

Given the diversity of bilinguals, research is rapidly moving away from simple questions regarding the presence or absence of a bilingual advantage, and instead is investigating when and where bilingualism makes a difference using a variety of tasks that examine differences not only between monolinguals and bilinguals, but also within heterogeneous bilingual groups. The current study uses a linguistic Stroop task (Stroop, 1935) to examine language control, and a non-linguistic Attention Network Test (ANT; Fan, McCandliss, Sommer, Raz \& Posner, 2002) to examine general cognitive control and attentional abilities in bilinguals immersed in their second language (L2) at various ages; this study aims to explore differences, if any, among these groups.

\section{Cognitive control in bilinguals}

Over the past two decades, there has been considerable interest in how bilingualism influences cognitive control. The seminal work of Bialystok et al. (2004) found that, relative to monolinguals, bilinguals show better interference suppression, and, while improved interference suppression argued for an inhibitory control advantage, the reaction time (RT) data showed that these benefits were broader in nature. Bilinguals showed faster RTs both in tasks that did and did not require interference suppression. Bialystok et al.'s (2008) work supported their previous findings, and extended them to show these bilingual advantages in both the nonlinguistic Simon task, as well as the linguistic Stroop task. This suggests that not just inhibitory processes, but also attentional processes, which could divert attention away from irrelevant information (Grundy, Chung-Fat-
Yim, Friesen, Mak \& Bialystok, 2017), benefit from bilingual experience.

Looking at attentional abilities specifically, Costa et al. (2008) examined monolinguals and bilinguals using the non-linguistic ANT task (Fan et al., 2002), in which RTs are measured as participants indicate the direction of a central target arrow while ignoring interference from flanking directional arrows. In addition, attentional cues assess Alerting, Orienting, and Executive Control networks (Fan, Gu, Guise, Liu, Fossella, Wang \& Posner, 2009). Again, bilinguals outperformed their monolingual counterparts. Particularly, bilinguals showed less interference from misleading information in incongruent trials, and, just as in Bialystok et al.'s (2004; 2008) studies, had faster global RTs. These observed bilingual advantages were credited to an improved Executive Control network. Supporting these findings, Marzecová, Asanowicz, Krivá, and Wodniecka (2013) found more efficient Executive Control networks for bilinguals, and greater accuracy in both congruent and incongruent trials; however, the expected global RT advantage was absent. Furthermore, while Pelham and Abrams (2014) found no differences in accuracy between monolingual and bilingual groups, they similarly reported less interference in the incongruent condition (i.e., better Executive Control) for bilinguals relative to monolinguals.

Although showing bilingual benefits, these studies also note that not all cognitive abilities are improved equally. Costa et al. (2008) found improved Alerting efficiency for bilinguals, and reason that, due to having to pay attention to two different language systems and switch between them, bilinguals are able to maintain a better state of alertness. An Alerting advantage, however, is not present in Costa et al.'s (2009) follow-up study. Both studies also fail to find a significant Orienting advantage, as do Hernández, Costa, Sebastián-Gallés, Fuentes, and Vivas (2010) and Marzecová et al. (2013). Such findings support Bialystok et al.'s (2008) observations that different aspects of cognitive control are affected differentially. Thus, it appears that while bilinguals enjoy some processing advantages, these do not extend to all cognitive abilities. Such findings are particularly relevant in light of recent debates.

Focusing on the young adult population, some researchers point out that not only are advantages not universal, but also that they are inconsistent (see Hilchey \& Klein, 2011; Hilchey et al., 2015). Examining non-linguistic studies of cognitive control, Hilchey and Klein (2011) note that, while there may be general processing advantages for young adult bilinguals, when inhibitory advantages are found, "they appear only briefly, early on, and dissipate very rapidly" (p.654). Showing this inconsistency across tasks is a series of experiments by Paap and Greenberg (2013). Testing young adult participants, the authors were unable to 
find consistent cognitive advantages for the bilingual group. Furthermore, the results showed little correlation in abilities across tasks. Kousaie and Phillips (2012a) also fail to find an advantage in the Stroop task for either group, and no differences between monolinguals and bilinguals in Stroop and flanker tasks (Kousaie \& Phillips, 2012b). Looking at these studies, what becomes clear is that the effects of bilingualism on cognitive control are varied, complex, and elusive.

\section{Bilingual tasks}

A number of reasons have been suggested for the mixed findings. One of the issues raised is the so-called "task impurity" problem (Miyake, Friedman, Emerson, Witzki, \& Howerter, 2000; Miyake \& Friedman, 2012; Valian, 2015). This essentially describes the impossibility of mapping one task to one component of cognitive control (see also Kroll \& Bialystok, 2013). For example, while the popular Stroop task (Stroop, 1935), in which the congruent condition is created by a text-colour match and the incongruent condition is created by a mismatch, has been used to measure inhibition, the task is not a measure of this process only. Responding correctly in the Stroop task requires not only inhibition of the irrelevant stimuli, but also processes such as attention, and while being a measure of cognitive control, the task's linguistic nature confounds cognitive control with reading processes. Because it is difficult to pinpoint the exact process required for a given task, it is also difficult to match tasks. As Valian (2015) points out, tasks that appear similar may not actually require the same processing abilities. She adds that other cognitive processes may be involved in between-group differences, and it is therefore difficult to know whether it is cognitive control differences or other factors that account for the results. Thus, it is difficult to say whether the tasks presently used are actual indicators of the cognitive processes they are meant to measure.

The difficulty of the task itself may also be a factor. Costa et al. (2009) were among the first to demonstrate that experimental conditions can also affect the outcome in non-linguistic tasks. The authors varied the proportion of congruent and incongruent ANT trials in their study, and found that in the low-conflict version of the task, the groups were fairly evenly matched. However, in the higher conflict version, the bilinguals surpassed the monolinguals. From this study and those that followed (e.g., Tao, Marzecová, Taft, Asanowicz \& Wodniecka, 2011), it appears that even bilingual young adults enjoy processing advantages that are not available to their monolingual counterparts, particularly in challenging environments.

For bilinguals, task effects were also shown by $\mathrm{Wu}$ and Thierry (2013). Bilinguals responded to ANT-like arrows, which were shown together with words in either a mixed-language context or a single-language context, thus creating a higher and lower level of difficulty, respectively. The authors found that, while RTs did not significantly differ between the blocks, there was a significant interaction between error rates and congruency, with their bilinguals making fewer errors in the mixed-language block compared to the single-language. The authors suggest that conflict resolution is easier in this morechallenging task. A similar effect of mixed- vs. singlelanguage blocks was shown by Sabourin and Vinerte (2015), who, using a bilingual Stroop task, showed that while single-language blocks in both languages yielded no differences in RTs between bilingual groups, differences between simultaneous and early bilinguals became apparent in the mixed-language block.

Considering issues like task impurity, leading to difficulty in examining which cognitive processes participants are using in behavioural tasks, and changes that come about as a result of manipulating task conditions, it becomes clear that there remains much work to be done in designing and implementing different tasks in cognitive control studies. Identifying the specific processes required for specific tasks, the locus of each process, and how these processes interact, continues to be an ongoing challenge for the field of bilingualism and cognitive control. Neurophysiological studies are instrumental in helping to resolve these issues, and works such as Abutalebi and Green (e.g., 2007; 2008) and Coderre et al. (2016), as well as meta-analyses such as Luk, Green, Abutalebi, and Grady (2012) have been able to shed light on some processes of cognitive control. However, findings at a behavioural level also should not be ignored, and, thus, challenges remain.

\section{Bilingual participants}

While we may not be able to control which processes participants use in tasks, we can identify characteristics of participants we examine. This too, however, has been raised as an issue in studies of bilingual cognitive control (see Kroll \& Bialystok, 2013). One topic specifically discussed is the definition of "bilingual". And as Luk (2015) asks, who are our monolinguals? Bilingual groups have been defined in many ways. Costa et al. (2008) used the language of the surrounding community. The authors acknowledge that this may introduce confounds, but reason that, as all participants were university students in the same program, these other factors would not impact their performance in the ANT. In Bialystok et al.'s (2008) study, bilinguals were defined as ones who used English and another language (which could be one of 24 languages) daily. Many participants were immigrants, but all were proficient in English and had similar degrees of formal education. Similiarly, Paap and Greenberg's 
(2013) bilingual group consisted of native and non-native speakers of English (including immigrants), with various language pairs.

While these approaches allowed for a categorical division of participants into monolinguals and bilinguals, they did not consider the heterogeneous nature of the bilingual group itself. Participants are not only differentiated by their status as "bilingual" and "monolingual"; both groups also vary in a host of other characteristics that should be taken into account (Luk, 2015). Different bilingual experiences may affect the development of cognitive control and therefore affect the outcomes of tasks, such as is shown by the very different results across studies. Taking this heterogeneity into consideration, studies have examined not only monolingual-bilingual differences, but also differences within bilingual groups themselves.

\section{Age of second language acquisition and cognitive control}

One within-group factor that reflects the amount of bilingual experience is the age of acquisition (AoA) of the second language. With respect to AoA, it is possible that the prolonged practice of being bilingual alters anatomical structures (Bialystok et al., 2012; Abutalebi \& Green, 2008), which in turn has consequences for behaviour. Becoming bilingual early in life also has another obvious advantage: namely, early bilinguals have spent a greater length of time being bilingual, and therefore have more experience in managing two language systems. However, thorough investigations of AoA effects on cognitive control, particularly in young adults, remain sparse.

In a study focusing specifically on AoA effects, Tao et al. (2011) used the ANT to examine a group of ChineseEnglish bilinguals, classified as Early or Late acquirers based on their age of arrival in Australia. Supporting the bilingual cognitive control advantage, this study revealed better efficiency across networks for bilinguals compared to monolinguals, but when the AoA groups were compared, another finding emerged. While early bilinguals had faster overall RTs, it was the late bilingual group that showed improved Alerting and Executive Control (no Orienting advantage was found.)

A subsequent study of a mix of Polish-L2 and SlovakL2 bilinguals (3 possible L2s; AoA was defined by age of first exposure to the L2) by Marzecová et al. (2013), however, found the opposite. While still finding an Executive Control network advantage for all bilinguals, it was the early bilinguals who showed the greatest Alerting network efficiency. Furthermore, no global RT advantages were found; an RT advantage only became apparent on conflict trials. The authors suggest that bilingualism does impact both conflict resolution and monitoring, but possibly in an independent manner. This suggests that, while becoming bilingual at any age confers an advantage, there are differences in the type of advantage. Results were interpreted as dissociating the conflict resolution advantage from a monitoring advantage.

A different approach to AoA was taken by Luk, de Sa, and Bialystok (2011), who examined the onset age of active bilingualism in young adults using a flanker task. Onset age of bilingualism is defined as the age at which a bilingual started using both languages daily, with earlier onset resulting in a greater length of bilingual experience. Once again, while an overall bilingual advantage was found, the early bilingual group showed the greatest ability to suppress interference in the flanker task. Interestingly, there were no differences in cognitive control ability between the late and monolinguals groups.

Pelham and Abrams (2014) also investigated Early and Late bilinguals, basing these groups of English-Spanish bilinguals on the age at which they became fluent in their L2. This study examined both lexical access and cognitive control, and found that while the bilinguals had a lexical access disadvantage in a picture naming tasks (supporting Bialystok et al., 2008), they showed better performance in an ANT task relative to monolinguals. However, there were no significant differences between the two bilingual groups. Therefore, the advantages for the bilingual groups were attributed to the constant use of two languages, rather than to the length of time spent being bilingual.

Finally, Yow and $\mathrm{Li}$ (2015) investigated non-immigrant young adult early Mandarin-English bilinguals who had learned both languages before the age of 7 in either home or school contexts. These bilinguals differed in the age at which they began to learn the $\mathrm{L} 2$, and consequently had a different balance of use and proficiency for each language. They were tested on the Stroop task, a number-letter task, the flanker task, and an n-back task, and while the latter two tasks showed no impact of bilingualism, there were strong bilingual effects for the Stroop and number-letter tasks, with earlier AoA groups showing better interference suppression than those who acquired their L2 later.

From these five studies, one point quickly becomes apparent: while all studies examine the same variable, AoA, they all take a very different approach to defining it. Tao et al. (2011) use the age of immigration, Marzecová et al. (2013) use the age of first exposure, Luk et al. (2011) use onset of active bilingualism, Pelham and Abrams (2014) define groups by the age of becoming fluent, while Yow and $\mathrm{Li}$ (2015) treat AoA as a continuous variable based on when the bilinguals began to learn the $\mathrm{L} 2$. All are measures of AoA, and all lead to some positive outcomes, yet all do not lead to the same outcomes, nor should we expect them to. The experience of becoming bilingual as a result of immigration may be quite different from becoming fluent in a second language in your country of 
birth. Despite these differences, AoA appears to be an important factor.

\section{The current studies}

The current study uses both the linguistic Stroop (Stroop, 1935) and the non-linguistic ANT (Fan et al., 2002) tasks, and aims to examine the conditions under which bilingualism impacts linguistic and non-linguistic cognitive control in a sample of age- and education-matched non-immigrant young adult bilinguals and monolinguals. Rather than focusing just on the presence or absence of bilingual advantages, we specifically focus on the differences among groups. We take into consideration the heterogeneous nature of bilingualism: the bilingual sample is subdivided into AoA groups. Based on previous work, we operationalize AoA as the Age of Immersion (AoI): the age at which an individual became immersed in his or her second language in a school setting through a French immersion programme (i.e., the student is exposed to French not only in a language class, but also takes courses such as, for example, mathematics, science, and history in French, thereby being immersed in the French language for a significant part of his/her day) in the case of sequential bilinguals, or, in the case of simultaneous bilinguals, through daily significant exposure to both languages through primary caregivers (e.g., one English and one French speaking parent). Immersion has been identified as a factor in linguistic cognitive control by Heidlmayr, Moutier, Hemforth, Courin, Tanzmeister, and Isel (2014), yet few studies have explored this variable. Also, while previous studies have looked at early bilinguals, often defined as those who have acquired a L 2 before the age of 6 or 7 (Tao et al., 2011), following previous work (Sabourin, Brien \& Burkholder, 2014; Sabourin \& Vinerte, 2015), we further subdivide this group into Simultaneous and Early bilinguals, an approach rarely taken in the existing literature. To minimize the amount of variability, all our participants and their parents are born in Canada, speak only English and French, and are undergraduate students in the same university. Studies such as Bialystok et al. (2008) and Paap and Greenberg (2013) included a large variety of language pairs, which greatly increases the variability of the sample, which may in part contribute to inconsistent results. Following Luk's (2015) recommendations, we take the linguistic environment of our participants into consideration. Our participants are drawn from the Ottawa community, in the predominantly Englishspeaking province of Ontario, and are English-dominant. Our simultaneous bilinguals grow up in bilingual households (self-report from our language background questionnaire), and all other participants are frequently exposed to both English and French in the community.

Given the heated debate and current controversy surrounding the bilingual advantage hypothesis, we seek to identify what, if any, advantages in linguistic cognitive control are conferred by bilingualism. We hypothesize that the impact of bilingualism will be holistic in nature, and earlier AoI will result in shorter RTs and greater accuracy, as well as less interference, in both the ANT and Stroop tasks; however, bilingual effects will be modulated by the task conditions. Although not as great as for earlier bilinguals, even late bilinguals are predicted to show reduced interference in both tasks. Furthermore, based on our previous work, different attentional networks in the ANT are predicted to be enhanced for each AoI group. While the results of this study cannot be generalized across different cognitive control tasks, they may give an insight into language and general cognitive control in bilinguals.

\section{Experiment 1. The Stroop task}

The Stroop task (Stroop, 1935) presents participants with colour terms, which can be either congruent or incongruent with the colour of the ink. In the congruent condition, the written word matches the colour of the ink (the word red written in red ink), while the incongruent condition consists of a mismatch (the word red written in green ink). Typically, it takes longer to name colours in the incongruent condition, and this increase in naming latency has been termed the "Stroop effect".

Of the theories put forth to explain this phenomenon, the more widely accepted is one of selective visual attention (e.g., Bialystok et al., 2008; Coderre, Van Heuven \& Conklin, 2013; Kousaie \& Phillips, 2012a, $2012 b$ ). Under this view, a greater amount of attentional resources are required for colour naming, a controlled process that requires cognitive effort, than for the process of reading, which is automatic and requires little effort for a proficient, literate individual (LaBerge \& Samuels, 1974). These processes occur in parallel, and it is specifically the direct competition between diverging word and colour information that is the source of interference and the Stroop effect (MacLeod \& MacDonald, 2000). The current study adheres to the view that decreased magnitudes of Stroop interference are indicative of better inhibition and attentional resource allocation.

In addition to interference, the Stroop task can also be used to examine facilitation. While the incongruent condition presents conflicting information that must be resolved, in the congruent condition, the information given by the written word and the visual colour are the same. Bialystok et al. (2008) found that, relative to neutral trials, words presented in congruent trials (the word red written in red ink) showed faster naming latencies. It was suggested that this is the result of the written word and the ink colour converging to give the same correct response. While this is not a process of cognitive control, it does provide insight into language processing in general. 
Table 1. Participant information. Average values provided for age and proficiency data.

\begin{tabular}{llllllll}
\hline \hline Group & & & Age of L2 & Age of & English & French \\
Exposure & Immersion & Proficiency (\%) & Proficiency (\%) & $\begin{array}{l}\text { Self-rated } \\
\text { proficiency (/20) }\end{array}$ \\
\hline Simultaneous Bilingual & 23 & $18.5(0.8)$ & $0.0(0.0)$ & 0 & $80.8(9.5)$ & $60.1(19.4)^{* *}$ & $18.7(1.3)$ \\
Early Sequential Bilingual & 20 & $19.1(1.7)$ & $4.4(1.2)$ & $1-6$ & $80.4(10.7)$ & $45.3(16.6)$ & $14.8(2.9)$ \\
Late Sequential Bilingual & 22 & $18.4(1.7)$ & $7.5(3.9)$ & $8+$ & $84.3(13.9)$ & $36.3(27.7)$ & $9.8(5.8)$ \\
Functional Monolingual & 22 & $18.5(2.7)$ & $8.9(2.4)$ & N/A & $83.4(10.7)$ & $1.4(10.7)$ & $5.0(3.3)$ \\
\hline \hline
\end{tabular}

** Due to the relatively greater difficulty of the French Cloze task, all participants scored lower on this task. However, there are no significant differences between the scores of native speakers of French (matched sample tested separately) and the Simultaneous group $(p=1.00)$, and no significant differences between the Early and Late groups $(p=1.00)$, indicating that these groups are matched in objective measures of proficiency.

\section{Methods}

\section{Participants}

A total of 87 participants (6 male) between the ages of 17 and 27 took part in this study. Participants self-reported as right-handed, with normal or corrected-to-normal vision, normal colour vision, and no history of brain injury. Participants were recruited through the university's Integrated System of Participation in Research and received 1 point credit. Participants completed a detailed language background questionnaire (Sabourin, Leclerc, Lapierre, Burkholder \& Brien, 2016), in which they indicated their ages of exposure and immersion in both English and French, their use of both languages (and any others), and assessed their proficiency in each. In addition, they completed an English Cloze task (Brown, 1980) and French Cloze task (Tremblay, 2011). Detailed participant information is provided in Table 1.

All participants were native speakers of English and all reported using English on a daily basis. Based on the language background questionnaire, participants were divided into three bilingual AoI groups: Simultaneous bilinguals, immersed in both English and French from birth as a result of being part of a bilingual household (often, with one Anglophone and one Francophone parent), Early Sequential bilinguals (hereafter abbreviated as 'early'), immersed in French between the age of 1 and 6, and Late Sequential bilinguals ("Late"), immersed in French after the age of 8 . In addition, we included a Functional Monolingual ("Monolingual") control group. As part of their Canadian education, these individuals have taken core French classes (approximately 45 minutes of French instruction per day) up until about grade 10, but had never been immersed in French, and currently have little to no written or oral communication proficiency.

\section{Stimuli and design}

An English Stroop task and a French Stroop task were programmed using Neurobehavioral Systems Presentation v.14.9 software. Stimuli were presented on a 19-inch screen. Participants used a Cedrus RB-830 button box equipped with 8 buttons to respond. Six buttons in the upper row were labeled with appropriate colours (red, yellow, green on the left side, and black, silver, white on the right side) and designated as response buttons, and two buttons in the lower row were unlabeled and designated as buttons to press to continue the experiment after instructions or a self-timed break. A manual rather than verbal response device was chosen because it has been shown that bilinguals are at disadvantage for lexical retrieval (e.g., Bialystok et al., 2008; Pelham \& Abrams, 2014). A study by Kousaie and Phillips (2012a) showed that there were no bilingual advantages in the Stroop task; however, the participants in this study named colours verbally, which may have cancelled out any bilingual advantages. Therefore, the current study uses a button box.

The stimuli used were the words RED, YELLOW, GREEN, BLACK, SILVER, WHITE for English, and ROUGE, JAUNE, VERT, NOIR, ARGENT, BLANC for French. Additionally, the high-frequency words TABLE, CHAIR, FORK, SPOON, HOUSE, and BOOK were included as control items for English, and CHAISE (chair), ARBRE (tree), SOURIS (mouse), STYLO (pen), MAISON (house), and LIVRE (book) were included as control items for French. Words were presented in capital letters in size 60 Times New Roman font in the centre of the screen.

Three separate blocks were created, containing Control (e.g., TABLE, presented in any colour), Congruent (e.g., RED presented in red font), and incongruent (RED presented in green font) trials. An English block containing English Control, English Congruent, and English Stroop items intermixed; a French block containing French Control, French Congruent, and French Stroop items intermixed; and a mixed block containing English and French Control, Congruent, and Incongruent items intermixed were created.

\section{Procedure}

Participants completed the English, French, and Mixed blocks of the task. Blocks were counterbalanced for 


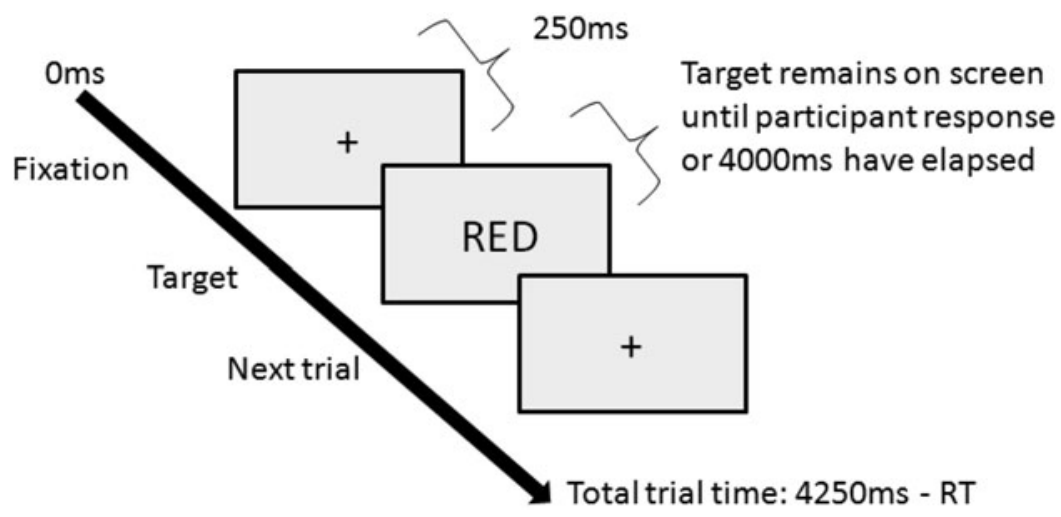

Figure 1. Stroop task procedure.

order of presentation, and self-timed breaks appeared between blocks. The initial block was preceded by 15 practice trials, and 5 practice trials appeared before each subsequent block. Participants were instructed to press a button to indicate the colour of the word on the screen, and it was emphasized that they should focus on the colour of the word. Each trial began with a fixation cross in the middle of the screen for $250 \mathrm{~ms}$. This was followed by the onset of the target. Once the target appeared on screen, it remained there until the participant responded or $4000 \mathrm{~ms}$ had elapsed. Participants took approximately 10 minutes to complete the 3 blocks, containing 25 trials each of Control, Congruent, and Incongruent trials, in each of the single-language blocks, and the 25 trials of each condition in each language in the mixed-language block, for a total of 300 trials in the experiment. See Figure 1 for a sample trial.

\section{Results}

Of the 87 participants, three participants (1 Monolingual, 1 Simultaneous, 1 Early) were excluded from the analysis as a result of a data recording malfunction. For the remaining participants, data from missed or incorrect trials was removed, and individual participant responses that were 2.5 standard deviations above or below the mean for that participant were also removed. Mean RT for each participant and each item was calculated, and box plots were conducted for each condition. No items were revealed as outliers, but the average RTs of 1 Simultaneous and 1 Late bilingual were considered as outliers, and their data was subsequently removed. Participant removal accounted for the removal of a total of $5.7 \%$ of the data. Because of high accuracy ( $>96 \%$ correct trials) and no significant group differences (all $p$ 's $=1.00$ ), further analysis of accuracy was not conducted, and only RT data from the remaining 82 participants was analysed.

\section{English Stroop task}

In the English language task, RT data reveals an effect of Congruency $(\mathrm{F}(2,124)=68.61, p<.001)$, showing the expected interference (indicated by slower responses in the Incongruent condition compared to the Control) and facilitation (indicated by faster responses in the Congruent condition compared to the Control) for English colour-word stimuli. Interference and facilitation were present for all native speakers of English, but there was no interaction between Congruency and AoI $(p=.61)$, indicating that responses were not influenced by their L2 French experience (Figure 2A).

\section{French Stroop task}

In the French language task, RT data once again reveals an effect of Congruency $(\mathrm{F}(2,149)=54.51, p<.001)$, and an interaction between Congruency and $\operatorname{AoI}(\mathrm{F}(6,149)=$ $2.23, p=.041)$. This interaction is driven by faster RTs for the Monolingual group compared to the Simultaneous, Early, and Late groups (a difference of $72.1 \mathrm{~ms}, 64.2 \mathrm{~ms}$, and $44.2 \mathrm{~ms}$, respectively), and the difference reflects the Monolingual group's lack of experience with the French language seen by the fact that they show the smallest Stroop effect (Figure 2B).

\section{Mixed Stroop task}

A repeated-measures ANOVA of the RTs in the Mixed Stroop task shows that just as in the English and the French tasks, there is an effect of Congruency $(\mathrm{F}(2$, $156)=116.78, p<.001)$, with significant interference for Incongruent trials (mean difference $=37.96 \mathrm{~ms}, p<.001$ ), and significant facilitation for Congruent trials (mean difference $=50.78 \mathrm{~ms}, p<.001)$, as well as a trend toward Language $\mathrm{x}$ AoI interaction $(\mathrm{F}(6,154)=1.98, p=.072)$. Importantly, a significant 3-way interaction between Congruency, AoI, and Language $(\mathrm{F}(6,156)=3.69$, $p=.003$ ) was found. To interpret this interaction, separate 

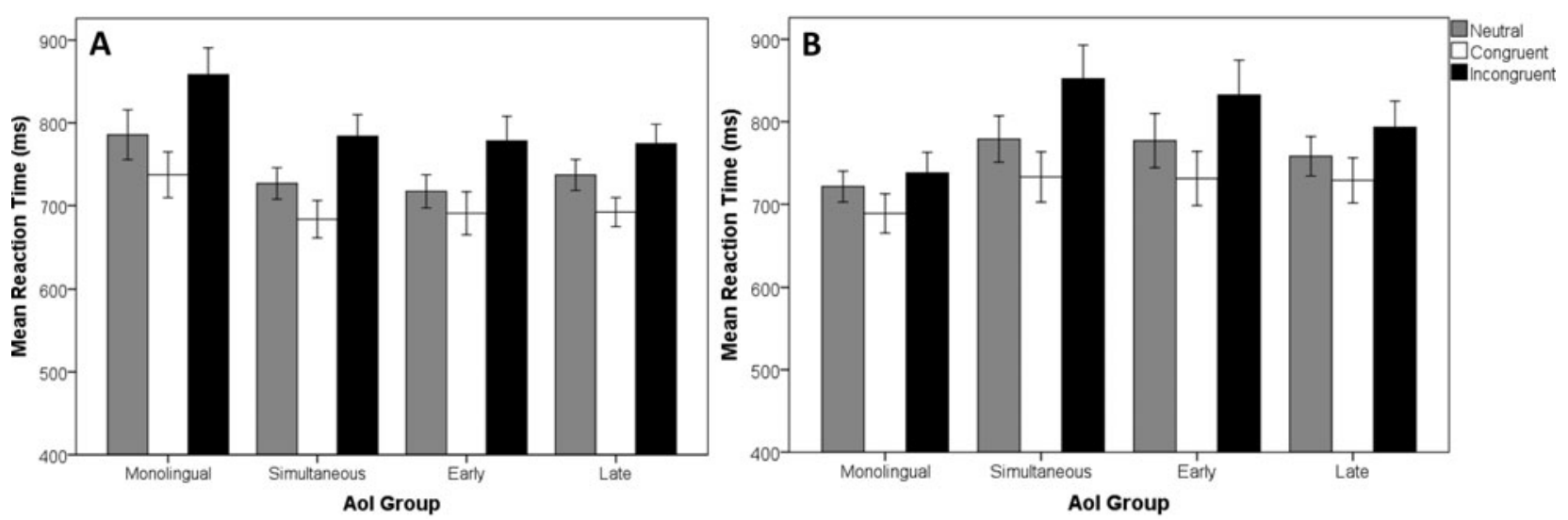

Figure 2. Mean Reaction Times by Condition for AoI Groups. Error bars represent Standard. Error. A represents the English Stroop task, and B represents the French Stroop task.

Congruence $\mathrm{x}$ Language ANOVAs were conducted for each AoI group.

For the Simultaneous group, there was an effect of Congruency $(\mathrm{F}(2,37)=25.53, p<.001)$, indicating the expected Interference (mean difference $=55.56 \mathrm{~ms}$, $p<.001)$ and Facilitation $(62.03 \mathrm{~ms}, p<.001)$ effects. In addition, there was an interaction between Congruency and Language $(\mathrm{F}(2,36)=5.54, p=.010)$, which was examined to further to reveal only Interference (mean difference $=82.82 \mathrm{~ms}, p<.001$ ), but no Facilitation (mean difference $=7.18 \mathrm{~ms}, p=1.00$ ) for English stimuli, and Facilitation (mean difference $=35.17 \mathrm{~ms}, p=.03$ ), but no Interference (mean difference $=28.30 \mathrm{~ms}, p=.24$ ) for French stimuli.

Similarly, for the Early group, there was an effect of Congruency $(\mathrm{F}(2,35)=39.51, p<.001)$, with significant Interference (mean difference $=47.09 \mathrm{~ms}, p=.007$ ) and Facilitation effects (mean difference $=60.40 \mathrm{~ms}, p<.001$ ). In addition, there was a trend towards an interaction between Congruency and Language $(\mathrm{F}(2,35)=2.94$, $p=.068)$. This trend was explored, and revealed a pattern opposite of that for the Simultaneous group: Facilitation (mean difference $=86.30 \mathrm{~ms}, p<.001)$, but no Interference (mean difference $=29.30 \mathrm{~ms}, p=0.40$ ) for English stimuli, and Interference (mean difference= $68.87 \mathrm{~ms}, p=.001$ ), but only a trend toward Facilitation (mean difference $=24.51 \mathrm{~ms}, p=.091$ ) for French stimuli were found for the Early group.

For the Late group, there was again an effect of Congruency $(\mathrm{F}(2,35)=20.83, p<.001)$, with significant Interference (mean difference $=27.35 \mathrm{~ms}, p=.018$ ) and Facilitation $(39.05 \mathrm{~ms}, p=.012)$ effects. However, no interaction between Congruency and Language was found $(p=.110)$. This same pattern was found for the Monolingual group, which showed a main effect of Congruency $(\mathrm{F}(2,35)=33.42, p<.001)$, demonstrating both Interference (mean difference $=37.32 \mathrm{~ms}, p=.001$ ) and Facilitation (mean difference $=49.61 \mathrm{~ms}, p=.001$ ), but these showed no interaction with Language $(p=.125)$. These results are shown in Figure 3.

\section{Experiment 2. The Attention Network Test}

Although the Stroop task allows us to measure cognitive control, because it is a language task, it confounds language processing with more general cognitive processes. To examine non-linguistic cognitive control, our study also included the ANT task, which is designed to measure three attentional networks, as proposed by Posner and Petersen (1990): Alerting, which is the achieving and maintaining of an alert state; Orienting, which is the selection of specific elements of the input; and Executive Control, which is involved in monitoring and conflict resolution (Fan et al., 2002). The researchers show that that these networks are relatively independent (Fan, McCandliss, Fossella, Flombaum \& Posner, 2005), yet function in an integrated manner (Fan et al., 2009). This demonstrates that attention is not a single mechanism, but rather, it is made up of several components that interact.

To test these networks, Fan et al. (2002) developed the ANT task, which combines timing and four cue conditions in addition to a flanker task consisting of Control, Congruent, and Incongruent arrows. The Control condition is formed by a single arrow pointing left or right and flanked by dashes on either side. In the Congruent condition, the central arrow appears with flankers pointing in the same direction, while, in the Incongruent condition, the flankers point in the opposite direction of the central arrow, thus creating a conflict situation. Participants respond by indicating the direction of the central target arrow. The target arrows are preceded by either a fixation cross only (a No-cue condition), or a fixation cross and one of 3 different attentional cues: Central cue, where the asterisk appears over the fixation cross; Double cue, where an asterisk appears above and below the fixation cross simultaneously; or a Spatial cue, which can be an asterisk 


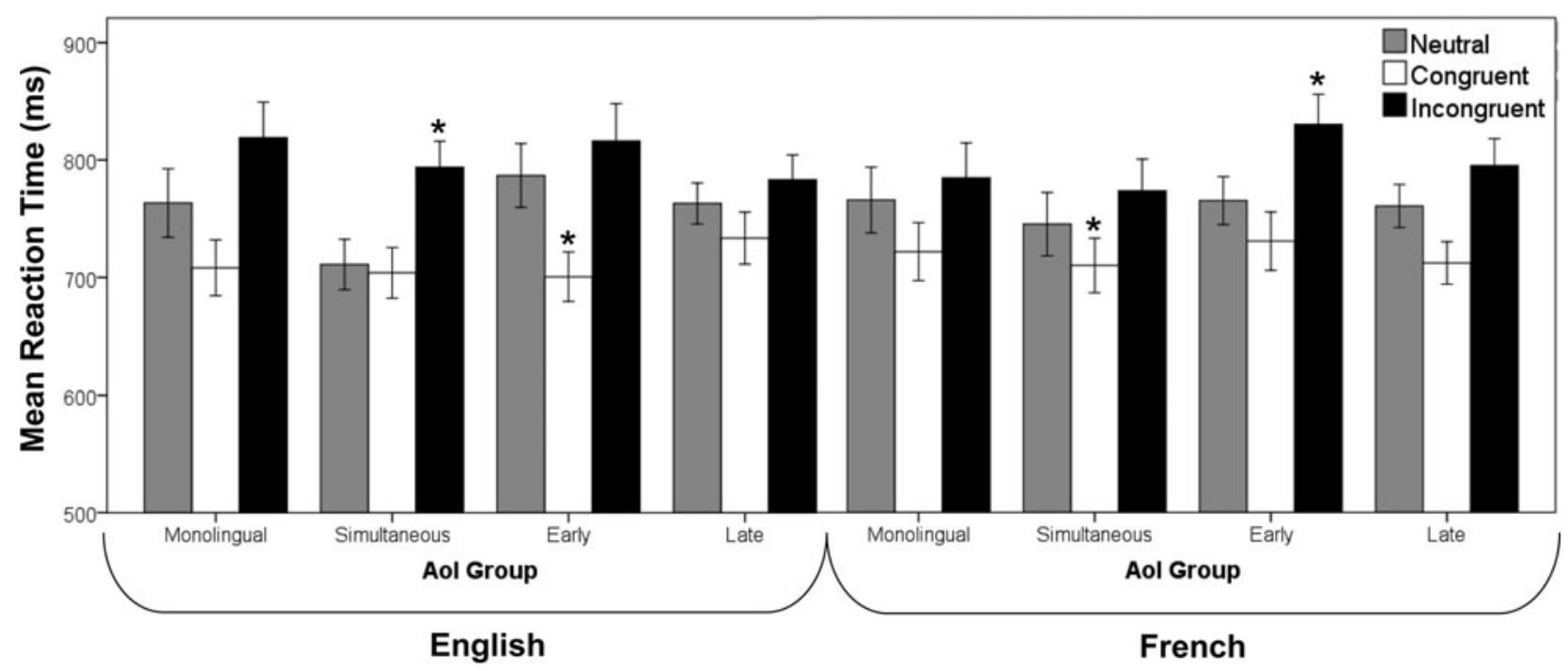

Figure 3. Mean Reaction Time by Condition for AoI Groups in the Mixed Stroop Task. RTs for English stimuli are shown on the right and RTs for French stimuli are shown on the left.

appearing above or below the fixation cross, indicating the location of the subsequent target.

To determine Alerting network efficiency, the RT difference between trials that contain a Double cue before the onset of the arrows and trials that contain no preceding cue is calculated. The Orienting network efficiency is examined by comparing trials in which a congruent Spatial cue indicates the exact location of the target and trials where this spatial information is absent due to the cue simply appearing in the centre of the screen, and thus providing no spatial information. It is calculated as the RT difference between trials with Central and Spatial cues.

The final network is Executive Control, and is calculated as the difference in RTs between Congruent and Incongruent conditions. Not only does this task allow for an examination of specific attentional processes, but it also provides a non-linguistic measure of cognitive control. While the Executive Control network conditions make the task similar to the Stroop in its ability to examine inhibition, the addition of cues makes this test a better measure of attention measures such as Alerting and Orienting. In controlling two simultaneously-active languages, bilinguals must focus attention on the target language while inhibiting the non-target one, and, thus, it is hypothesized that bilingualism will particularly enhance this network.

\section{Methods}

\section{Participants}

The same population and grouping as for the Stroop task presented in Section 3 were used for the ANT task. Refer to Table 1 for full participant details.

\section{Stimuli and design}

An ANT task was programmed using Neurobehavioral Systems Presentation v.14.9 software. Stimuli were presented using a PC desktop computer with a Windows Vista operating system, on a 19-inch screen. Participants used a Cedrus RB-830 button box to respond: a button was designated for the left and the right arrow response, and a third button was designated as a "continue" button to be pressed after instruction screens and self-timed breaks. To minimize cognitive demand of responses, the left and right response buttons were placed on the left and right side, respectively.

In the current study, the stimuli used were left-pointing and right-pointing arrows, and asterisks $\left(^{*}\right)$ were used as cues. Arrows could appear in either the Control, Congruent, or Incongruent conditions, either above or below a central fixation cross, combined with any of the four Cue types. An ANT task with 288 trials was created and separated into two identical blocks, each consisting of 144 trials. Trials were randomized, but each block contained equal numbers of Control, Congruent, and Incongruent trials, and equal numbers of each of the 4 Cues. Arrow congruency and Cue types are shown in Figure 4A.

\section{Procedure}

The ANT trial began with a fixation cross at the centre of the screen for $250-1500 \mathrm{~ms}$, followed by a $100 \mathrm{~ms}$ presentation of the cue. The cue disappeared, and $250 \mathrm{~ms}$ later the target appeared on screen until the participant responded or $2000 \mathrm{~ms}$ had elapsed. Participants responded by pressing either the left or the right button to indicate the direction of the arrow they had seen. The next trial began immediately following the button 

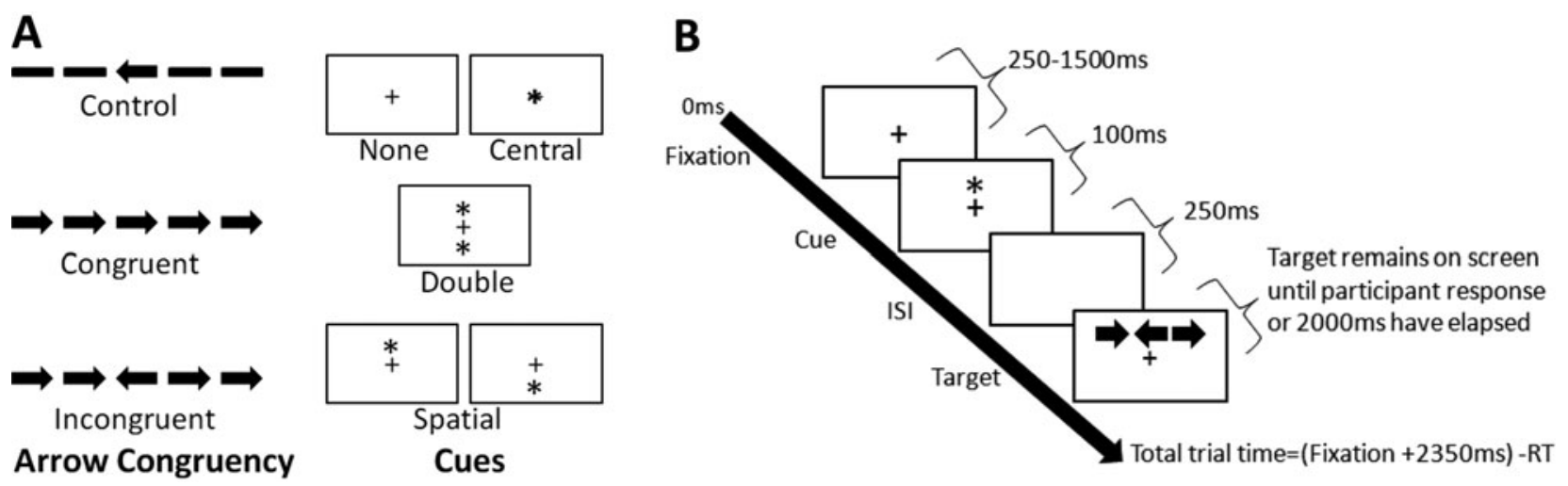

Figure 4. The ANT Task. Arrow Congruency and Cue Conditions are shown in panel A and a sample trial is given in panel B.

press. Each block was preceded by 15 practice trials, and blocks were separated by a self-timed break. Throughout the blocks, trials were randomized using Presentation's randomization command, and the 288 experimental trials and practice blocks took approximately 20 minutes to complete. See Figure 4B for a sample trial with a Spatial Cue and an Incongruent target.

\section{Results}

For the 87 participants, data from missed or incorrect trials was removed and individual participant responses that were 2.5 standard deviations above or below the mean for that participant were removed. Mean RT for each participant was calculated, and box plots were conducted for each condition. Average RTs revealed 3 participants as significant outliers (1 Monolingual, 1 Simultaneous and 1 Late bilingual) and their data was subsequently removed. Participant removal accounted for the removal of a total of $3.4 \%$ of the data. Because of high accuracy ( $>95 \%$ correct trials) and no significant group differences (all $p$ 's $=1.00$ ), further analysis of accuracy was not conducted, and only RT data from the remaining 84 participants was analysed.

\section{General analysis}

A Cue (None, Central, Double, Spatial) x Congruency (Neutral, Congruent, Incongruent) $x$ AoI ANOVA revealed the expected Congruency effect $(\mathrm{F}(2$, 116) $=399.45, p<.001$ ), demonstrating facilitation for the congruent compared to the neutral condition (mean difference $=44.70 \mathrm{~ms}, p=.017$ ) and interference for the incongruent compared to neutral condition (mean difference $=64.03 \mathrm{~ms}, p<.001)$. Furthermore, there was an effect of Cue $(\mathrm{F}(3,196)=223.00, p<.001)$, and effects showing Alerting (None vs. Double Cue; mean difference $=38.91 \mathrm{~ms}, p<.001)$ and Orienting (Central vs. Spatial Cue; mean difference $=22.61 \mathrm{~ms}, p<.001$ )
There was also an interaction between Congruency and $\operatorname{AoI}(\mathrm{F}(6,116)=2.58, p=0.036)$, and results also revealed an interaction between Cue and Congruency $(\mathrm{F}(6,425)$ $=14.28, p<.001)$. To interpret these interactions, we followed Fan et al.'s (2002) ANT analysis method, which looked at the individual effect sizes for the different networks and examined each attentional network separately.

\section{Effect sizes}

To examine the interaction between Congruency and AoI, an analysis of the effect sizes for each attentional network was conducted. The analysis of the Executive Control network revealed significant differences between the groups $(\mathrm{F}(3,78)=2.86, p=.042)$, and planned pairwise comparisons showed that this effect is driven particularly by the Simultaneous group's increased efficiency compared to the Monolingual group (mean difference $=21.00 \mathrm{~ms}, p=.044$ ). However, there were no between group differences for the Alerting $(\mathrm{F}=2.15$, $p=.101)$ and Orienting $(\mathrm{F}<1, p=.480)$ networks. These network effects are shown in Figure 5.

Typically, in an analysis of the ANT task, the Executive Control network is calculated as the difference between the congruent and incongruent conditions; however, these are collapsed across all cue types. The interaction between Cue and Congruency prompted us to further analyse the effects of Cue and to attempt to disentangle the effects of each factor.

\section{Cue effects}

Although there was no significant interaction between the Cue, Congruency, and AoI, the interaction between Cue and Congruency prompted our final analysis. We examined the patterns for the Executive Control network, calculating the effect size for the network for each separate cue type. When looking at Executive Control in the non-cued condition (None), there were no differences between groups $(\mathrm{F}<1, p=.914)$. Similarly, 


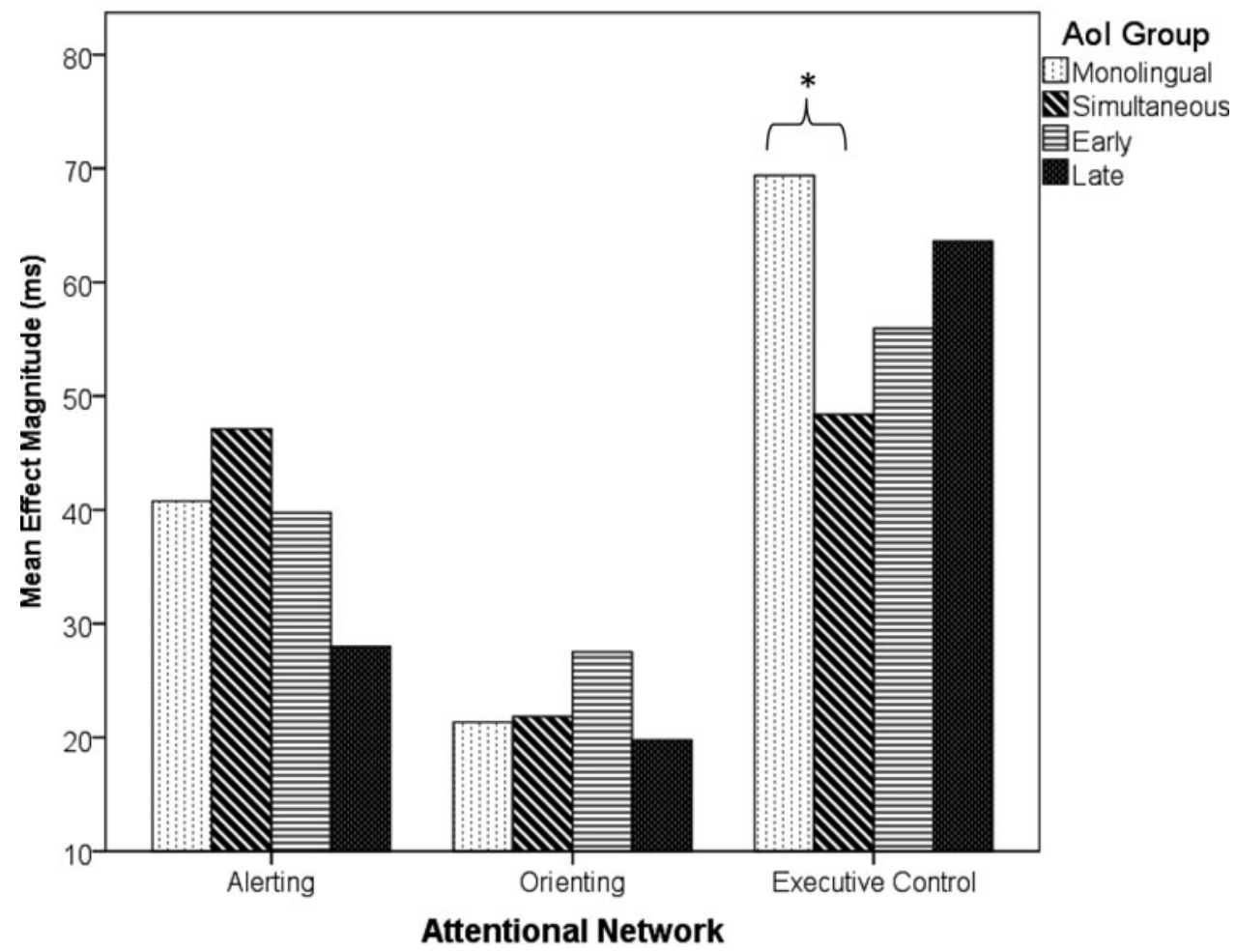

Figure 5. Attentional Network Effects by AoI Group. Calculated network effects shown. For Executive Control network, lower effect magnitude indicates greater network efficiency, while for the Alerting and Orienting networks, greater efficiency is indicated by greater effect magnitude.

no differences were seen with the Central cue $(\mathrm{F}=1.81$, $p=.152$ ). However, when looking at Executive Control in the Double cue and Spatial cue conditions, there are significant differences between the groups $(\mathrm{F}(3,78)=3.25$, $p=.026$ and $\mathrm{F}(3,78)=2.87, p=0.042$, respectively). Simultaneous bilinguals benefit most in both conditions, showing less interference compared to the Late group (mean difference $=28.94 \mathrm{~ms}, p=.037$ ) in the Double cued condition, and compared to the Monolingual group (mean difference $=30.98 \mathrm{~ms}, p=.036$ ) in trials with a Spatial cue. These effects are shown in Figure 6.

\section{Discussion and conclusions}

The current study investigated linguistic and nonlinguistic cognitive control in young adult bilinguals, a group whose results have been particularly inconsistent in the literature. We conducted two experiments, using the Stroop task and the ANT, in bilinguals of different AoA's, which we operationalized as the age at which the individual became immersed in their L2. To minimize variability, all participants were native speakers of English with French as the other language. All participants were from the same region and matched for age and education. A complex pattern of results emerged, showing that bilingualism is not a detriment to cognitive ability, and particularly demonstrating that task characteristics and AoI may play a role in linguistic processing, and linguistic and non-linguistic cognitive control.

The linguistic Stroop task in Experiment 1 shows an interaction between language processing and control, and demonstrates differential effects based on the complexity of the task for young adult bilinguals. Examining each language separately, there is no apparent advantage for individuals who are more bilingual as a function of having been immersed in their L2 earlier in life. Such results are consistent with previous findings demonstrating no bilingual effects in the Stroop task (Kousaie \& Phillips, 2012a, 2012b). Looking at Figure 2B, it appears that the monolingual group has an advantage in processing the French stimuli; however, it is unlikely that this reflects improved cognitive control abilities. Although this group has had some exposure to their L2 French, their proficiency in the language is low. Thus, it is more likely that the faster RTs reflect a lack of familiarity with French that leads to the monolingual group not processing French stimuli as language, but as simple colour items. Although they show some interference, this is considerably smaller than their bilingual counterparts, suggesting that the Monolingual group's processing of L2 French is not automatic. 


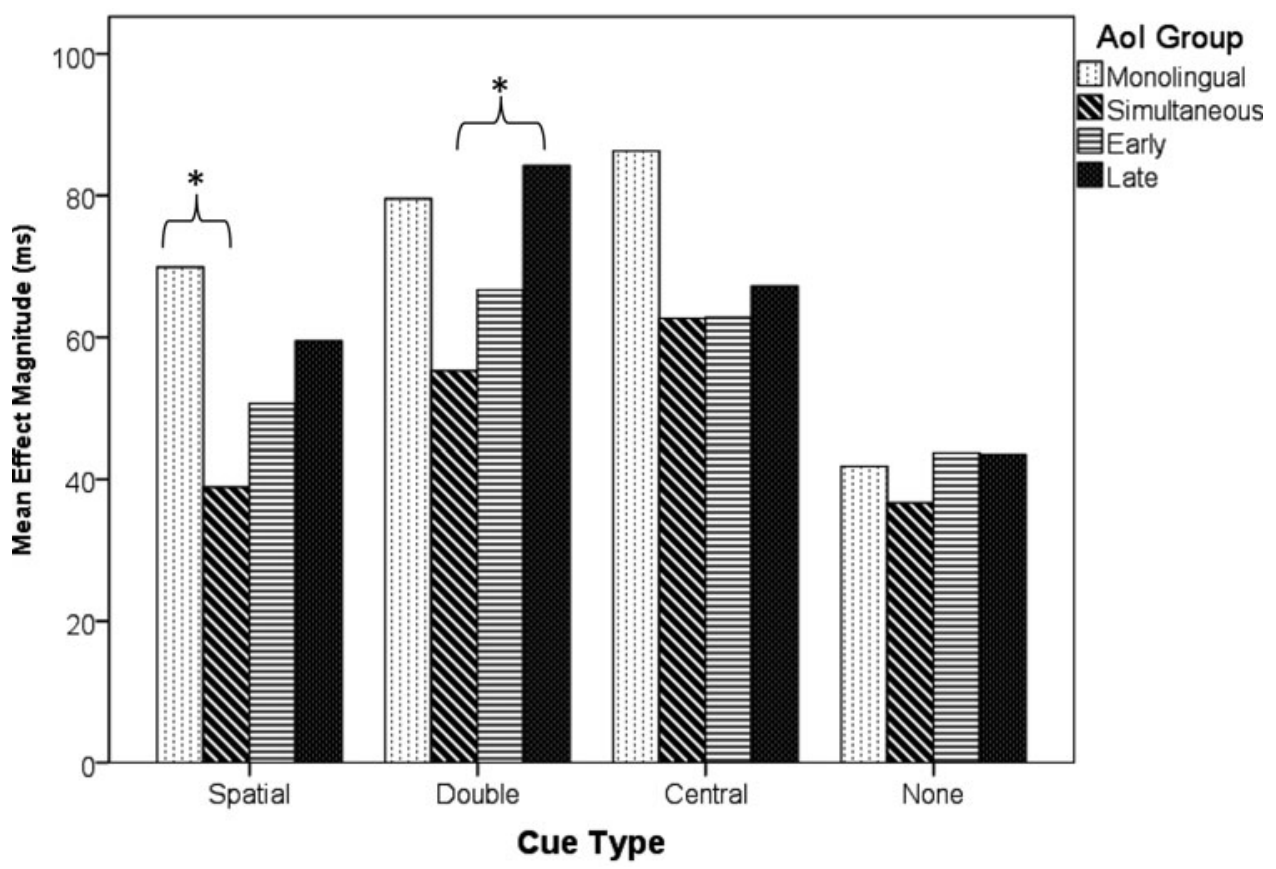

Figure 6. Executive Control Network Efficiency by Cue Type for AoI Groups. Calculated network effects shown for each cue type. Lower effect magnitude indicates greater network efficiency.

Examining processing when both languages are mixed, current results echo our previous work (Sabourin \& Vinerte, 2015), showing that it is particularly when the task challenge is increased that we see an influence of bilingualism. Such task effects have been previously reported (e.g., Costa, 2008; 2009), and have been attributed to young adults being at their prime in terms of cognitive processing. This leaves little room for improvement caused by bilingualism, and may contribute to the lack of observed bilingual effects when looking at performance in single language conditions such as those used here. As Costa et al. (2009) and Wu and Thierry (2013) show, bilingual effects become more apparent when the task difficulty is increased.

When looking at the mixed Stroop task, particularly noteworthy is the opposite pattern of results for Early and Simultaneous bilinguals: while the Early bilinguals show only Facilitation in English and Interference in French, Simultaneous bilinguals show only Interference in English and Facilitation in French. The English-dominant Early bilinguals thus show a strong ability to manage the interference in their native language, while being able to use facilitating cues to speed performance. Although highly skilled in French, they are unable to control the interference from stimuli in their L2. For the linguisticallybalanced Simultaneous group, their greater experience with French allows them to use facilitating cues to boost performance in French, while their experience of living in an English-dominant environment may result in greater interference from this language. It is important to note that in the case of the Simultaneous bilinguals, these differences cannot be attributed to differences between the first and the second language, as both languages are acquired at the same time. The results of the Early and Simultaneous bilinguals are in contrast with those of the Late bilinguals and Monolinguals, with the latter two groups showing similar patterns of performance (also observed by Luk et al., 2011), but no differences or advantages, suggesting that it is particularly earlier immersion that leads to these language processing differences. Although group differences warrant further investigation, Experiment 1 leads us to conclude that among other factors, task complexity is an important factor in determining performance.

While the Stroop task shows different patterns of results for each of the AoI groups, these results demonstrate differences in language control and processing among bilinguals with different L2 experience, but do not speak to general cognitive control abilities. Indeed, Hilchey and Klein (2011) specifically do not include the Stroop task in their analysis due to its close relationship to language, and say that in order to demonstrate the benefits of bilingualism on general cognitive control, tasks that aren't language-driven are required.

Examining the non-linguistic ANT task and attentional networks in Experiment 2, while no effect of bilingualism on the Alerting and Orienting networks was found, Simultaneous bilinguals showed significantly improved efficiency compared to their monolingual counterparts. 
Effectively, there is an Executive Control advantage for the most bilingual participants (those immersed in two languages from birth) relative to the least bilingual participants (those who, while having some exposure to French, have never been immersed and have low proficiency). Such findings are in line with previous studies (e.g., Costa et al., 2008; Marzecová et al., 2013), which also found Executive Control advantages for bilingual groups. With respect to the Early and Late bilingual groups, the pattern of results suggests that the Executive Control networks of these individuals also benefit relative to the Monolingual group, as was shown by Pelham and Abrams (2014); although in the current study, these effects do not reach significance.

Various mechanisms have been suggested to explain the Executive Control advantage for bilinguals, with a common one being conflict monitoring and resolution (Costa et al., 2008; 2009; Pelham \& Abrams, 2014). According to this account, bilinguals' constant need to monitor for and resolve linguistic conflict in the environment leads to better cognitive control in tasks involving conflict. Bilinguals' heightened monitoring in conflict situations is suggested to "spill over" to nonconflict situations. Another has been that of attentional control (e.g., Bialystok et al., 2004; 2008, Grundy et al., 2017), which proposes that bilingualism improves one's ability to manage attention when faced with complex and changing tasks. The engagement and disengagement of attention is more efficient, leading to bilingual advantages (see Grundy et al., 2017). The results of the current study support the latter mechanism, as seen by our examination of cue effects separately from congruency.

The current study found no Alerting or Orienting attentional network effects; however, this may be the result of these network calculations collapsing cues across all congruencies. In an attempt to disentangle cue effects from congruency, the Executive Control network was examined under different cue conditions, and this examination leads us to conclude that bilingualism is most beneficial to attentional mechanisms. Simultaneous bilinguals benefited from the Double cue and the Spatial cue, the two cue types specifically associated with the alerting and orienting of attention. The Double cue alerts participants to the imminent onset of the target, engaging their attentional resources, while the Spatial cue signals the exact location of the upcoming target, engaging attentional resources and directing them toward the relevant task. It may be that this group's experience in tuning attention from one language to the other allows them to take advantage of such facilitating cues in nonlinguistic tasks as well. Looking at the effect size among other bilingual groups relative to the monolingual one, distinct benefits of the attentional cues can also be seen for the Early group, with some benefits for the Late group, suggesting that while not as significant as for the Simultaneous group, these groups also benefit from facilitating attentional cues more than monolinguals.

Interestingly, there are no differences among the groups when no cue is present, suggesting two possibilities for the cued vs. non-cued trials: 1) In the non-cued trials, without engaging attention, all groups are functioning at peak efficiency, and therefore there are no differences in among them; or 2) by introducing the cues, distractors that divert attention away from the target are also introduced, thereby increasing the difficulty of the task. Under these latter conditions, it may be the bilingual participants who are best able to tune attention to minimize distraction and use this additional information to complete the task, leading to improved Executive Control. However, the additional information provided by the cues distracts the monolingual group, and this distraction leads to a less efficient Executive Control network. To determine this with certainty, however, further investigation is needed.

Taken together, the current results suggest that there may be an advantage to being immersed in the L2 at a younger age, for both language and domain-general cognitive processing. Furthermore, they show that while Simultaneous and Early bilinguals are immersed in both languages from a very young age, their underlying language and cognitive processes are different. Thus, grouping them together, as is commonly done, may contribute to the lack of differences seen between monolinguals and bilinguals in some of the literature. For example, combining the two in a linguistic Stroop task may result in Interference and Facilitation effects canceling each other out, leading to apparent null effects.

Furthermore, the results suggest that not only the task but also the task analysis need to be taken in to consideration in bilingual cognitive control studies. Hilchey and Klein (2011) and Hilchey et al. (2015) consider a number of flanker tasks in their investigation of the validity of bilingual advantages, and conclude that there are inconsistencies among the results. However, as seen in the current study, no effects are apparent in the non-cued condition (essentially, the standard flanker task). Interestingly, our results also suggest caution when interpreting ANT network effects. In a psychometric assessment, MacLeod, Lawrence, McConnell, Eskes, Klein, and Shore (2010) note that the reliability of the Alerting and Orienting networks is low, and suggest that these networks may not be independent. Our analysis, while not finding any Alerting and Orienting effect differences between the bilingual groups, only noted differences of the alerting and orienting cue types when cue type and congruency were disentangled.

To fully understand the differences among bilinguals and tasks, it may be necessary to use a larger participant sample, more sophisticated statistical measures, as well as measures more sensitive than behavioural ones, such as event-related brain potentials. While our participants are 
divided into four categorical groups, Luk and Bialystok (2013) suggest that this may not accurately reflect the bilingual experience and recommend examining bilinguals on a continuum. We agree that bilingualism is not a strictly categorical variable, and acknowledge that one of the limitations of the current study is the sample size, which restricted us to the use of categorical groups. With this in mind, we chose four points on the spectrum which best represented the linguistic situation of our participants; however, future studies should address the full continuum.

A further limitation of the current study was the statistical approach. Baum and Titone (2014) and van Heuven and Coderre (2015) suggest reconsidering current statistical approaches such as ANOVAs. Although broadly used, ANOVAs necessarily limit analysis to group means of a few categorical variables, making it impossible to account for all the variability of a bilingual sample. As Baum and Titone (2014) observe, researchers are therefore moving more towards regression analyses and mixed effects modelling. While this may be a superior statistical approach, the size of the current sample and the data collection procedures limited us to the use of ANOVAs. Future studies would benefit from mixedeffects modelling to better understand the differences among bilinguals.

Finally, while behavioural data shows the end-point of processing, it is not sensitive to the underlying mechanisms throughout processing. Thus, any possible underlying differences are obscured. While the two groups look similar on the surface, the underlying pathways that allow each group to arrive at the response may be vastly different. Even if the cognitive resources used are similar, there may be differences in underlying processing and in how and when each of these resources is employed. Indeed, when behavioural measures have shown no between-group differences, an examination of neural processes reveals the opposite (e.g., Kousaie \& Phillips, 2012b).

Furthermore, based on the work of Green and Abutalebi (2013), we believe that the linguistic environment of our bilingual participants also contributes to their performance. Ottawa, while being English dominant, is a bilingual city, and switching between English and French are not uncommon. According to the Adaptive Control Hypothesis, it could be classified as a dense code-switching environment. The Mixed Stroop task mimics our participants' environment, and, as their cognitive processes may be adapted to such conditions, it is only when the two languages are mixed that any effects are seen. The Adaptive Control Hypothesis may also explain the Simultaneous group's ANT results. This model predicts that, under dense code-switching conditions, opportunistic planning will be significantly improved, which may have allowed the
Simultaneous group to take advantage of the attentional cues to facilitate their response to the targets. For the monolingual group, however, these cues functioned only as distractors, decreasing the efficiency of cognitive control. Such processing is also compatible with Bialystok and colleagues' proposal that attentional mechanisms are what particularly benefit from the bilingual experience.

Although our study shows some advantages of bilingualism, these patterns are complex and inconsistent, agreeing with Hilchey and Klein's assessment of young adult bilinguals. However, our results are in line with Valian's (2015) observation that, when found, bilingual effects tend to be positive. In no condition were monolinguals at a true advantage over bilinguals. Part of the inconsistency in results may come from a lack of sensitivity of the method. In both Experiment 1 and Experiment 2, increasing the task difficulty led to observable effects for the Simultaneous and Early vs. Monolingual groups, who are the most different in terms of their AoI, but it failed to show any significant differences for the Late bilingual group (although some differences were noticeable in RT and effect size patterns). Thus, to see these more fine-grained differences, future studies should combine behavioural and neurophysiological techniques, as the latter may be capable of assessing processes obscured in behavioural tasks alone.

\section{References}

Abutalebi, J., \& Green, D. (2007). Bilingual language production: The neurocognition of language representation and control. Journal of Neurolinguistics, 20, 242-275.

Abutalebi, J., \& Green, D. W. (2008). Control mechanisms in bilingual language production: neural evidence from language switching studies. Language and Cognitive Processes, 23, 557-582.

Baum, S., \& Titone, D. (2014). Moving toward a neuroplasticity view of bilingualism, executive control, and aging. Applied Psycholinguistics, 35, 857-894.

Bialystok, E., Craik, F., Klein, R., \& Viswanathan, M. (2004). Bilingualism, aging, and cognitive control: evidence from the Simon task. Psychology and Aging, 19(2), 290-303.

Bialystok, E., Craik, F., \& Luk, G. (2008). Cognitive control and lexical access in younger and older bilinguals. Journal of Experimental Psychology: Learning, Memory, and Cognition, 34(4), 859-873.

Bialystok, E., Craik, F., \& Luk, G. (2012). Bilingualism: consequences for mind and brain. Trends in Cognitive Sciences, 16(4), 240-250.

Brown, J. D. (1980). Relative merits of four methods for scoring cloze tests. Modern Language Journal, 64, 311-317.

Costa, A., Hernández, M., \& Sebastián-Gallés, N. (2008). Bilingualism aids in conflict resolution: Evidence from the ANT task. Cognition, 106, 59-86.

Costa, A., Hernández, M., Costa-Faidella, J., \& SebastiánGallés, N. (2009). On the bilingual advantage in conflict 
processing: Now you see it, now you don't. Cognition, 113, 135-149.

Coderre, E. L., van Heuven, W. J. B., \& Conklin, K. (2013). The timing and magnitude of Stroop interference and facilitation in monolinguals and bilinguals. Bilingualism: Language and Cognition, 16(2), 420-441.

Coderre, E. L., Smith, J. F., van Heuven, W. J. B., \& Horwitz, B. (2016). The functional overlap of executive control and language processing in bilinguals. Bilingualism: Language and Cognition, 19 (3), 471-488.

de Bruin, A., Treccani, B., \& Della Sala, S. (2015). Cognitive advantage in bilingualism: an example of publication bias? Psychological Science, 26 (1), 99-107.

Della Sala, S., \& Grafman, J. (Eds.). (2015). Bilingualism Forum [Special section]. Cortex, 73, 330-377.

Fan, J., Gu, X., Guise, K. G., Liu, X., Fossella, J., Wang, H., \& Posner, M. I. (2009). Testing the behavioral interaction and integration of attentional networks. Brain and Cognition, 70, 209-220.

Fan, J., McCandliss, B. D., Fossella, J., Flombaum, J. I., \& Posner, M. I. (2005). The activation of attentional networks. Neuroimage, 26(2), 471-479.

Fan, J., McCandliss, B. D., Sommer, T., Raz, A., \& Posner, M. I. (2002). Testing the efficiency and independence of attentional networks. Journal of Cognitive Neuroscience, 14(3), 340-347.

Green, D. W. (1998). Mental control of the bilingual lexicosemantic system. Bilingualism: Language and Cognition, $1,67-81$.

Green, D.W., \& Abutalebi, J. (2013). Language control in bilinguals: the adaptive control hypothesis. Journal of Cognitive Psychology, 25(5), 515-530.

Grundy, J. G., Chung-Fat-Yim, A., Friesen, D. C., Mak, L., \& Bialystok, E. (2017). Sequential congruency effects reveal differences in disengagement of attention for monolingual and bilingual young adults. Cognition, 163, 4255 .

Heidlmayr, K., Moutier, S., Hemforth, B., Courin, C., Tanzmeister, R., \& Isel, F. (2014). Successive bilingualism and executive functions: The effect of second language use on inhibitory control in a behavioural Stroop Colour Word task. Bilingualism: Language and Cognition, 17(3), 630 645.

Hernández, M., Costa, A., Sebastián-Gallés, N., Fuentes, L. J., \& Vivas, A. B. (2010). The impact of bilingualism on the executive control and orienting networks of attention. Bilingualism, 13(3), 315-325.

Hilchey, M. D., \& Klein, R. M. (2011). Are there bilingual advantages on nonlinguistic interference tasks? Implications for the plasticity of executive control processes. Psychonomic Bulletin \& Review, 18, 625-658.

Hilchey, M. D., Saint-Aubin, J., \& Klein, R. M. (2015). Does bilingual exercise enhance cognitive fitness in traditional non-linguistic executive processing tasks? In J. Schwieter (Ed.), The Cambridge handbook of bilingual processing. Cambridge: Cambridge University Press.

Kousaie, S., \& Phillips, N. A. (2012a). Aging and bilingualism: Absence of a "bilingual advantage" in Stroop interference in a nonimmigrant sample. Quarterly Journal of Experimental Psychology, 65(2), 356-369.
Kousaie, S., \& Phillips, N.A. (2012b). Conflict monitoring and resolution: Are two languages better than one? Evidence from reaction time and event-related brain potentials. Brain Research, 1446, 71-90.

Kroll, J.F., \& Bialystok, E. (2013). Understanding the consequences of bilingualism for language processing and cognition. Journal of Cognitive Psychology, 25(5), 497514.

Kroll, J.F., Bobb, S., \& Hoshino, N. (2014). Two languages in one mind: bilingualism as a tool to investigate language, cognition, and the brain. Current Directions in Psychological Science, 23(2), 159-163.

Kroll, J.F., Bobb, S.C., \& Wodniecka, Z. (2006). Language selectivity is the exception, not the rule: Arguments against a fixed locus of language selection in bilingual speech. Bilingualism: Language and Cognition, 9(2), 119135.

LaBerge, D., \& Samuels, S. J. (1974). Toward a theory of automatic information processing in reading. Cognitive Psychology, 6, 293-323.

Luk, G. (2015). Who are the bilinguals (and monolinguals)? Bilingualism: Language and Cognition, 18(1), 35-36.

Luk, G., de Sa, E., \& Bialystok, E. (2011). Is there a relation between onset age of bilingualism and enhancement of cognitive control? Bilingualism: Language and Cognition, 14(4), 588-595.

Luk, G., \& Bialystok, E. (2013). Bilingualism is not a categorical variable: Interaction between language proficiency and usage. Journal of Cognitive Psychology, 25, 605621.

Luk, G., Green, D. W., Abutalebi, J., \& Grady, C. (2012). Cognitive control for language switching in bilinguals: A quantitative meta-analysis of functional neuroimaging studies. Language and Cognitive Processes, 27(10), 14791488 .

MacLeod, C. M., \& MacDonald, P. A. (2000). Interdimensional interference in the Stroop effect: uncovering the cognitive and neural anatomy of attention. Trends in Cognitive Sciences, 4(10), 383-391.

MacLeod, J. W., Lawrence, M. A., McConnell, M. M., Eskes, G. A., Klein, R. M., \& Shore, D. I. (2010). Appraising the ANT: Psychometric and theoretical considerations of the Attention Network Test. Neuropsychology, 24(5), 637-651.

Marian, V., \& Spivey, M. (2003). Competing activation in bilingual language processing: Within- and betweenlanguage competition. Bilingualism: Language and Cognition, 6(2), 97-115.

Marzecová, A., Asanowicz, D., Krivá, L., \& Wodniecka, Z. (2013). The effects of bilingualism on efficiency and lateralization of attentional networks. Bilingualism: Language and Cognition, 16(3), 608-623.

Miyake, A., Friedman, N. P., Emerson, M. J., Witzki, A. H., Howerter, A., \& Wager, T. D. (2000). The unity and diversity of executive functions and their contributions to complex "Frontal Lobe" tasks: A latent variable analysis. Cognitive Psychology, 41, 49-100.

Miyake, A., \& Friedman, N. P. (2012). The nature and organization of individual differences in executive functions: four general conclusions. Current Directions in Psychological Science, 21(1), 8-14. 
Paap, K. R., \& Greenberg, Z. I. (2013). There is no coherent evidence for a bilingual advantage in executive processing. Cognitive Psychology, 66(2), 232-258.

Pelham, S.D., \& Abrams, L. (2014). Cognitive advantages and disadvantages for early and late bilinguals. Journal of Experimental Psychology, Learning, Memory, and Cognition, 40(2), 313-325.

Posner, M.I., \& Petersen, S.E. (1990). The attention system of the human brain. Annual Review of Neuroscience, 13, $25-42$.

Sabourin, L., Brien, C., \& Burkholder, M. (2014). The effect of age of L2 acquisition on the organization of the bilingual lexicon: Evidence from masked priming. Bilingualism: Language and Cognition, 17(3), 542-555.

Sabourin, L., Leclerc, J-C., Lapierre, M., Burkholder, M., \& Brien, C. (2016). The Language Background Questionnaire in L2 Research: Teasing Apart the Variables. Proceedings of the Canadian Linguistic Association. Available online.

Sabourin, L., \& Vinerte, S. (2015). The bilingual advantage in the Stroop task: simultaneous vs. early bilinguals. Bilingualism: Language and Cognition, 18(2), 350-355.

Sanchez-Azanza, V.A., Lópes-Penadés, R., Buil-Legaz, L., Aguilar-Mediavilla, E., \& Adrover-Roig, D. (2017). Is bilingualism losing its advantage? A bibliometric approach. PLoSONE, 12(4): e0176151.

Stocco, A., Yamasaki, B., Natalenko, R., \& Prat, C. S. (2014). Bilingual brain training, a neurobiological framework of how bilingual experience improves executive function. International Journal of Bilingualism, 18(1), 6792.

Stroop, J.R. (1935). Studies of interference in serial verbal reactions. Journal of Experimental Psychology, 18, 643662.

Tao, L., Marzecová, A., Taft, M., Asanowicz, D., \& Wodniecka, Z. (2011). The efficiency of attentional networks in early and late bilinguals: The role of age of acquisition. Frontiers in Psychology, 2, 1-19.

Tremblay, A. (2011). Proficiency assessment standards in second language acquisition research: "Clozing" the gap. Studies in Second Language Acquisition, 33, 339-372.

Valian, V. (2015). Bilingualism and cognition. Bilingualism: Language and Cognition, 18(1), 3-24.

van Heuven, W. J. B., \& Coderre, E. L. (2015). A call for sophisticated statistical approaches and neuroimaging techniques to study the bilingual advantage. Cortex, 73, 330-331.

Wu, Y.J., \& Thierry, G. (2013). Fast modulation of executive function by language context in bilinguals. Journal of Neuroscience, 33(33), 13533-13537.

Yow, W. Q., \& Li, X. (2015). Balanced bilingualism and early age of second language acquisition as the underlying mechanisms of a bilingual executive control advantage: why variations in bilingual experience matter. Frontiers in Psychology, 6:164, 1-12. 